\title{
The synergistic effect of minocycline and azole antifungal drugs against Scedosporium and Lomentospora species
}

Fang Yang ${ }^{1,2}$, Yi Sun ${ }^{3}$ and Qiaoyun $\mathrm{Lu}^{4^{*}}$

\begin{abstract}
Background: This study was aimed to determine the potency of Minocycline (MIN) and azoles, including itraconazole (ITR), voriconazole (VOR) and posaconazole (POS) against Scedosporium and Lomentospora species.

Results: This study revealed that MIN exhibited no significant antifungal activity against any of the tested strains, whereas in vitro combination of MIN with ITR, VOR or POS showed satisfactory synergistic effects against 8 (80\%), 1 (10\%), and 9 (90\%) strains, respectively. Moreover, combined use of MIN with azoles decreased the minimum inhibitory concentration (MIC) range from $5.33-16 \mu \mathrm{g} / \mathrm{ml}$ to $1-16 \mu \mathrm{g} / \mathrm{ml}$ for ITR, from $0.42-16 \mu \mathrm{g} / \mathrm{ml}$ to $0.21-16 \mu \mathrm{g} / \mathrm{ml}$ for VOR, and from $1.33-16 \mu \mathrm{g} / \mathrm{ml}$ to $0.33-16 \mu \mathrm{g} / \mathrm{ml}$ for POS. Meanwhile, no antagonistic interactions were observed between the above combinations. The G. mellonella infection model demonstrated the in vivo synergistic antifungal effect of MIN and azoles.

Conclusions: The present study demonstrated that combinations between MIN and azoles lead to synergistic antimicrobial effects on Scedosporium and Lomentospora species, while showing a potential for overcoming and preventing azole resistance.
\end{abstract}

Keywords: Scedosporium, Lomentospora, Minocycline, Azole, Synergy, Antifungal effect, Resistance

\section{Introduction}

The incidence of fungal infections is increasing year by year. In particular, invasive fungal infections are increasingly becoming an important factor endangering human health and life [1]. Invasive fungal infections are predisposed to occur in immunocompromised patients as well as hospitalized patients with severe underlying diseases $[2,3]$. Scedosporium and Lomentospora species are an aggressive and conditional pathogen, causing a rare type of fungal infection, with only 370 cases reported worldwide up to 2007 [4]. The infection involves a variety of sites and is relatively difficult to be diagnosed clinically

\footnotetext{
*Correspondence: xyluqiaoyun@163.com

${ }^{4}$ Xiangyang Central Hospital, Affiliated Hospital of Hubei University

of Arts and Science, Xiangyang, Hubei, China

Full list of author information is available at the end of the article
}

because it shares a great similarity in clinical characteristics and histopathology with aspergillosis, infection with Fusarium, and other relatively common hyalohyphomycosis [5].

Azoles, including itraconazole (ITR), voriconazole (VOR) and posaconazole (POS), are an earlier class of drugs used to treat fungal infections [6]. According to epidemiological investigation, the prevalence of Scedosporium and Lomentospora species is increasing, posing a serious threat to the effectiveness of antifungal drugs [7]. Liu et al. showed that Scedosporium and Lomentospora species are highly resistant to antifungal drugs, highlighting the need for alternative treatment modalities [8]. A recent study showed that Lomentospora prolificans and Scedosporium apiospermum were well tolerated by POS in combination with terbinafine [9]. Moreover, combination antifungal therapy with VOR has 
been demonstrated to be a promising treatment option for invasive, Lomentospora prolificans infections [10]. Collectively, these observations indicate that combination therapy could be an important clinical treatment strategy for this kind of refractory infection.

Minocycline (MIN) is a semisynthetic broad-spectrum tetracycline antibiotic that displays a similar antibacterial spectrum with tetracycline. MIN can combine with tRNA to achieve antibacterial effect, while being the most potent antibacterial agent among the existing tetracycline antibiotics. Moreover, previous studies have demonstrated that Azoles (fluconazole) in combination with MIN is a potential approach for counteracting Candida albicans-Staphylococcus aureus dual-species biofilms [11]. Based on these facts, we propose that MIN may have a certain antifungal effect, and as an additive, it may enhance the efficacy of commonly used azoles against Scedosporium and Lomentospora species.

\section{Results}

In vitro interactions between MIN and azoles against Scedosporium and Lomentospora species

As presented in Table 1, MICs for MIN were $\geq 16 \mu \mathrm{g} / \mathrm{mL}$ for all strains, while MICs for ITR were $\geq 16 \mu \mathrm{g} / \mathrm{mL}$ for 8 out of the 16 strains and ranged from 2 to $8 \mu \mathrm{g} / \mathrm{mL}$ for the rest strains. On the contrary, both VOR and POS displayed a lower MIC, ranging between 0.25 and $4 \mu \mathrm{g} / \mathrm{mL}$ for 10 out of the 12 strains. In the meantime, all drugs had a MIC of $\geq 16 \mu \mathrm{g} / \mathrm{mL}$ for the two Lomentospora strains.

Table $1 \mathrm{MIC}$ and $\mathrm{FICl}$ results with the combinations of MIN and azoles against Scedosporium apiospermum

\begin{tabular}{|c|c|c|c|c|c|c|c|}
\hline \multirow[t]{3}{*}{ Strain } & \multicolumn{7}{|c|}{ MIC (ug/mL) for: } \\
\hline & \multicolumn{4}{|c|}{ Agent alone } & \multicolumn{3}{|c|}{ Combination $[\mathrm{A} / \mathrm{B}(\mathrm{ug} / \mathrm{mL})](\mathrm{FICl})$} \\
\hline & MIN & ITR & VOR & POS & MIN/ITR & MIN/VOR & MIN/POS \\
\hline $\begin{array}{l}\text { Scedosporium aurantiacum } \\
\text { (CBS 116910) }\end{array}$ & $\geq 16$ & 8 & 0.5 & 2 & $4 / 2(S)$ & $4 / 0.25(I)$ & $8 / 0.5(\mathrm{~S})$ \\
\hline $\begin{array}{l}\text { Scedosporium minutispora } \\
\text { (CBS 116911) }\end{array}$ & $\geq 16$ & 2 & 0.25 & 2 & $4 / 0.25(S)$ & $8 / 0.25(I)$ & $8 / 0.5(S)$ \\
\hline $\begin{array}{l}\text { Scedosporium boydii } \\
\text { (CBS 101.22) }\end{array}$ & $\geq 16$ & 2 & 0.25 & 2 & $8 / 1(I)$ & $16 / 0.25(I)$ & $4 / 0.5(\mathrm{~S})$ \\
\hline $\begin{array}{l}\text { Lomentospora prolificans } \\
\text { (CBS 467.74) }\end{array}$ & $\geq 16$ & $\geq 16$ & 16 & $\geq 16$ & $4 / 8(S)$ & $4 / 8(I)$ & $4 / 8(S)$ \\
\hline $\begin{array}{l}\text { Scedosporium dehoogii } \\
\text { (CBS 117406) }\end{array}$ & $\geq 16$ & 2 & 0.5 & 2 & $4 / 1(1)$ & $4 / 0.5(I)$ & $8 / 0.5(\mathrm{~S})$ \\
\hline $\begin{array}{l}\text { Scedosporium apiospermum } \\
\text { (CBS 116899) }\end{array}$ & $\geq 16$ & 4 & 0.25 & 2 & $4 / 1(S)$ & $4 / 0.25(I)$ & $8 / 0.5(S)$ \\
\hline $\begin{array}{l}\text { Scedosporium boydii } \\
\text { (1) }\end{array}$ & $>16$ & $>16$ & 1 & 2 & $8 / 1(S)$ & $8 / 0.25(I)$ & $8 / 0.5(\mathrm{~S})$ \\
\hline $\begin{array}{l}\text { Scedosporium boydii } \\
\text { (2) }\end{array}$ & $>16$ & 4 & 1 & 1 & $4 / 1(S)$ & $8 / 0.25(I)$ & $8 / 0.25(S)$ \\
\hline $\begin{array}{l}\text { Scedosporium boydii } \\
\text { (3) }\end{array}$ & $>16$ & $>16$ & 1 & 4 & $8 / 2(S)$ & $8 / 0.5(I)$ & $8 / 1(S)$ \\
\hline $\begin{array}{l}\text { Scedosporium apiospermum } \\
\text { (4) }\end{array}$ & $>16$ & $>16$ & 1 & 4 & $8 / 4(S)$ & $8 / 0.25(I)$ & 4/1(S) \\
\hline $\begin{array}{l}\text { Scedosporium apiospermum } \\
\text { (5) }\end{array}$ & $>16$ & $>16$ & 1 & 2 & $8 / 4(S)$ & $8 / 0.5(I)$ & $8 / 0.5(\mathrm{~S})$ \\
\hline $\begin{array}{l}\text { Scedosporium apiospermum } \\
\text { (6) }\end{array}$ & $>16$ & $>16$ & 0.5 & 2 & $8 / 4(S)$ & $4 / 0.5(1)$ & $8 / 0.5(\mathrm{~S})$ \\
\hline $\begin{array}{l}\text { Lomentospora prolificans } \\
\text { (7) }\end{array}$ & $>16$ & $>16$ & $>16$ & $>16$ & $16 / 16(I)$ & $16 / 16(I)$ & $16 / 16(I)$ \\
\hline $\begin{array}{l}\text { Scedosporium minutisporum } \\
\text { (8) }\end{array}$ & $>16$ & 8 & 1 & 2 & $8 / 2(S)$ & $8 / 0.25(S)$ & $8 / 0.5(\mathrm{~S})$ \\
\hline $\begin{array}{l}\text { Scedosporium apiospermum } \\
\text { (9) }\end{array}$ & $>16$ & $>16$ & 0.5 & 4 & $8 / 16(I)$ & 8/0.125(I) & $8 / 1(S)$ \\
\hline $\begin{array}{l}\text { Scedosporium boydii } \\
\text { (10) }\end{array}$ & $>16$ & 4 & 0.25 & 1 & 4/1(S) & 8/0.125 (I) & 8/0.25(S) \\
\hline
\end{tabular}

MIC is determined by the concentrations of drugs at which growth was completely inhibited. FICl results are shown in parentheses. S, synergistic effect (FICl of 0.5$)$; I, no interaction (indifference) $(0.5 \mathrm{FICl} 4)$ 
Combination of MIN with ITR, VOR, or POS exhibited synergistic activities against $12(75.00 \%), 1(6.25 \%)$, or 15 (93.75\%) strains of Scedosporium and Lomentospora species (Table 1). Notably, the combination of MIN and VOR only displayed a synergistic activity against strain Scedosporium minutisporum (8). Meanwhile, no antagonistic interactions were detected in each of the tested combinations.

\section{In vivo interactions between MIN and azoles against Scedosporium and Lomentospora}

To assess the synergistic effects of MIN and azoles in vivo, G. mellonella were infected with Scedosporium apiospermum, and the infected larvae were then treated with MIN and azoles. As shown in Fig. 1, treatment with MIN in combination with POS, ITR or VOR led to a survival rate of $25,35 \%$ or $30 \%$. Moreover, we observed that compared with Infected Control-No Treatment group, VOR treatment slightly increased larval survival, while POS or ITR alone did not significantly increase the survival. By contrast, administration with MIN combined with one of Azoles significantly prolonged larval survival time $(P<0.05)$. Together, these findings demonstrated the in vivo synergistic antifungal activity of MIN and azoles against G. mellonella, as evidenced by the increased larval survival.

Uninfected Control Group, wild type larvae without Scedosporium apiospermum infection; Uninfected Control-Saline Treatment, wild type larvae injected with saline; Infected Control-No Treatment Group, larvae infected with Scedosporium apiospermum receiving no treatment; POS Group, Scedosporium infected larvae treated with POS only; POS + MIN Group, Scedosporium infected larvae treated with POS in combination with MIN; ITR Group, Scedosporium infected larvae treated with ITR only; ITR + MIN Group, Scedosporium infected larvae treated with ITR in combination with MIN; VOR Group, Scedosporium infected larvae treated with VOR only; VOR + MIN Group, Scedosporium infected larvae treated with VOR in combination with MIN; POS: Chlorhexidine; ITR: Itraconazole; VOR: Voriconazole. The experiment was repeated thrice on different days. "P $P<0.05 ; " P<0.01 ;{ }^{* * * * *} P<0.0001$.

\section{Histopathological analyses}

We further performed a histopathological examination of the larvae. As depicted in Fig. 2, the formation of Scedosporium apiospermum spores and hyphae clusters in the infected tissues was detected in all the experimental groups. We observed that treatment with azoles alone resulted in a slight reduction in the number of visible fungal clusters relative to the Scedosporium group, while the number of visible fungal clusters was significantly decreased in the combination treatment groups as compared to the three azoles alone groups.

\section{Discussion}

Scedosporium and Lomentospora species are important and very recalcitrant conditional pathogens. While the risk factors for infection of Scedosporium and Lomentospora species include a history of immunodeficiency, trauma, and drowning, the infection mainly involves skin, eyes, lungs, brain, even the whole body $[4,12]$. Over the past decade, many trials have demonstrated that single azoles such as ITR, POS, VOR, and fluconazole exhibit certain activity against Scedosporium and Lomentospora species in vitro [13, 14]. The widespread use of azoles has led to a shift from azole-susceptible to azole-resistant infections. As a result, existing azoles are not ideal for the empirical treatment of infections with Scedosporium and Lomentospora species. Given the low antimicrobial activity of single drugs, combined use of multiple drugs may enhance their antimicrobial activities. Azole antifungal agents are commonly used in combination with other types of antifungal drugs in clinical practice, such as amphotericin B [15].

\section{Galleria mellonella survival rates}

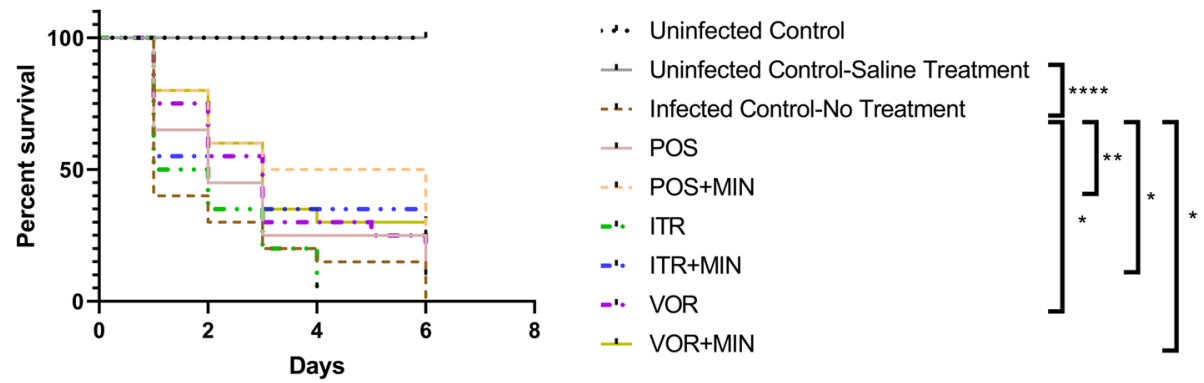

Fig. 1 G. mellonella survival rates 


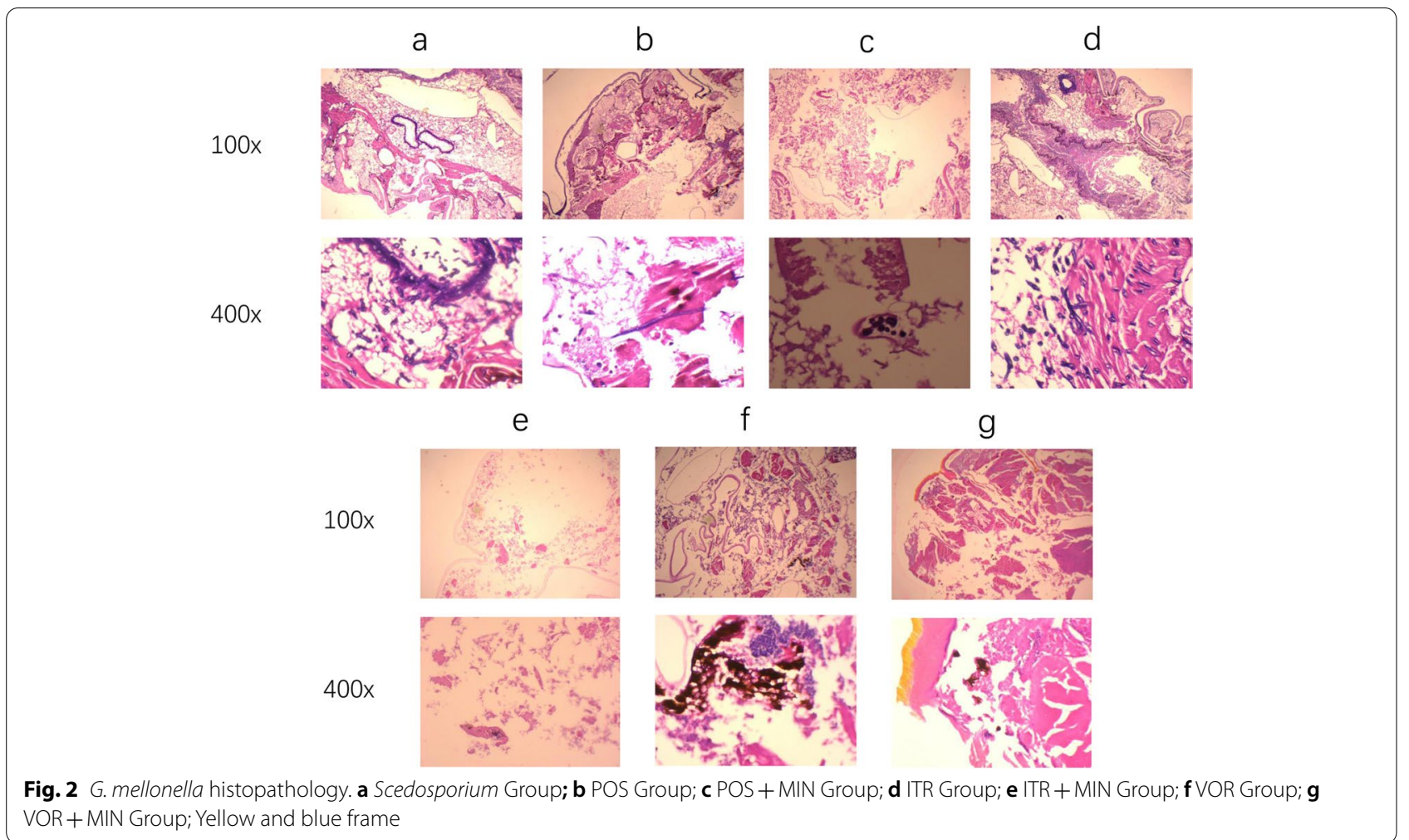

MIN has been used clinically as an antibiotic for many years since it was marketed. In recent years, MIN has gained an increasing attention with the research on its non-antimicrobial effect. Previous studies have shown that MIN reduces cell surface hydrophobicity and extracellular levels of 1,3-beta-D-glucan (1,3-BDG) in biofilms, revealing a mechanism underlying the inhibitory effect of MIN on Candida albicans [16]. Given that 1,3-BDG is the main component of fungal cell wall, it is suggested that MIN may possess an antifungal activity. Strikingly, MIN combined with low-dose fluconazole has been found to exert a synergistic antifungal effect [17, 18]. However, there have been few reports investigating the effect of MIN in combination with azoles against Scedosporium and Lomentospora species. In the present study, we examined the inhibitory effects of ITR, VOR, POS, and MIN alone as well as those of MIN in combination with azoles on Scedosporium and Lomentospora species.

Herein, a total of 16 isolates of Scedosporium and Lomentospora species were studied in vitro. While MIN alone showed no in vitro antifungal activity, MIN acted synergistically with ITR, VOR or POS against $8(80 \%), 1(10 \%)$, or $9(90 \%)$ isolates tested, showing promising synergistic effects between MIN and azoles against Scedosporium and Lomentospora species. In addition, we found that Lomentospora prolificans displays low susceptibility to current antifungal drugs, being consistent with the previous reports $[19,20]$. As listed in Table 1, we observed a significant decline in MICs of combination of MIN with ITR, VOR or POS against Scedosporium and Lomentospora species. The in vivo experiments provided more evidence that MIN acts synergistically with azoles against Scedosporium and Lomentospora species (Figs. 1 and 2). In agreement with a previous study showing that the presence of MIN increases susceptibility of pathogenic fungi to azoles, the present study did not identify any antagonism between MIN and azoles [21].

The combination of drugs is used mainly for increasing the efficacy of drugs or reducing their side effects. Fungal infections of the central nervous system (CNS) are characterized by the serious clinical manifestations as well as the difficulty in diagnosis and treatment, while Scedosporium infections affecting the CNS are relatively common [22, 23]. As a highly lipophilic molecule, MIN can easily enter cerebrospinal fluid and CNS through blood-brain barrier. Thus, it has potential for treating various CNS diseases [24, 25]. Overall, these observations may provide a theoretical basis for combined use of MIN and azoles in the treatment of fungal infections in CNS. 


\section{Conclusions}

In conclusion, MIN in combination with azoles may help to enhance the antifungal activities of azoles against Scedosporium and Lomentospora species, achieving a synergistic effect between MIN and azoles. Further studies need to be conducted for gaining insights into clinical impacts of the combination of MIN and azoles, as well as understanding clinical relevance of the in vitro data obtained in this study.

\section{Materials and methods Fungal strains}

A total of 16 strains were included in the study. Among them, Scedosporium aurantiacum (CBS 116910), Scedosporium minutispora (CBS 116911), Scedosporium boydii (CBS 101.22), Lomentospora prolificans (CBS 467.74), Scedosporium dehoogii (CBS 117406), and Scedosporium apiospermum (CBS 116899) were generously provided by Prof. Sybren de Hoog of Fungal Biodiversity Centre (CBS) in Netherlands. The other strains were purchased from Nanjing Institute of Dermatology, China, including Scedosporium boydii (1), Scedosporium boydii (2), Scedosporium boydii (3), Scedosporium apiospermum (4), Scedosporium apiospermum (5), Scedosporium apiospermum (6), Lomentospora prolificans (7), Scedosporium minutisporum (8), Scedosporium apiospermum (9), and Scedosporium boydii (10). The species identity of each isolate was determined based on combined morphologi$\mathrm{cal} /$ phenotypic characteristics and molecular sequencing of the internal transcribed spacer (ITS) ribosomal DNA (rDNA). Isolates were sub-cultured from frozen stocks on Sabouraud dextrose agar (SDA) prior to in vitro testing [26]. The authors declare that the ethical policies of the journal, as noted on the journal's author guidelines page, have been adhered to, and the study was approved by the ethical review committee. The procedures were conducted in accordance with the US National Research Council's guidelines for the Care and Use of Laboratory Animals.

\section{Antifungal agents}

All tested agents, including ITR, VOR, POS, and MIN, were obtained from Selleck Chemicals (Houston, TX, USA) in powder form and dissolved according to the manufacturer's protocol. The concentrations of tested agents were in the range from 0.5 to $64 \mathrm{mg} / \mathrm{L}$ for ITR, VOR and POS, or from 1 to $64 \mathrm{mg} / \mathrm{L}$ for MIN.

\section{In vitro susceptibility testing}

In vitro interaction between MIN and azoles against Scedosporium and Lomentospora species was analysed using a microdilution checkerboard technique [27]. Conidia were harvested from cultures, grown for 3 days on SDA and then suspended in sterile distilled water containing $0.03 \%$ Triton. Thereafter, harvested conidia were adjusted to $1-5 \times 10^{6} \mathrm{CFU} / \mathrm{ml}$ with a hemocytometer and then diluted 100 times with RPMI1640 broth containing $0.165 \mathrm{M}$ MOPS ( $\mathrm{pH} 7.0$ ) according to the M38-A2 reference standard. Serial dilutions of tested agents were prepared by dilution with RPMI 1640. For preparation of test microplates, $50 \mu \mathrm{l}$ of each concentration of azoles (ITR, VOR and POS) was added to columns 2 to 8 , while $50 \mu \mathrm{l}$ MIN was applied to rows 2 to 8 . In each microplate, while row 1 and column 1 contained the azoles and MIN alone, respectively, column 9 was a drug-free well serving as the growth control. One hundred microlitre of fungal inoculum was applied to 96 -well plates for a $72 \mathrm{~h}$ of incubation in ambient atmosphere at $35^{\circ} \mathrm{C}$, and the data were read visually. MIC values were defined as the lowest concentration of drugs at which the growth was completely inhibited. To assess the interaction outcomes, FICI value was calculated as follows: (MIC of Drug A in combination/MIC of Drug A alone) + (MIC of Drug $\mathrm{B}$ in combination/MIC of Drug $\mathrm{B}$ alone). The interaction of combined drugs was interpreted as FICI $\leq 0.5$ for synergy, $0.5<\mathrm{FICI}<4.0$ for no interaction, or $\mathrm{FICI} \geq 4$ for antagonism. All the tests were performed in triplicate.

\section{In vivo cytotoxicity assay}

G. mellonella larvae were divided into nine groups ( $N=20$ per group): control group, Uninfected ControlSaline Treatment group, Infected Control-No Treatment group, MIN group, ITR group, VOR group, POS group, combined ITR and MIN group, combined VOR and MIN group, and combined POS and MIN group. We used several inoculation concentrations for infection. Since lower or higher concentrations resulted in low or high mortality in the control group, we used the optimal concentration for the observation. All nine groups except the control group and Uninfected Control-Saline Treatment group were injected with $S$. apiospermum (CBS 116899). G. mellonella was pricked into the needle only, but not injected. The experimental group was injected with $10 \mathrm{ul}$ of S. apiospermum (CBS 116899 ) solution at a concentration of $1 \times 10^{7}$. S. apiospermum was injected into the last right proleg. Antifungal agents or a control solution $(1 \mu \mathrm{g}$ per larvae; drug concentration $=200 \mathrm{mg} / \mathrm{L}$ ) was introduced after the area was cleaned with an alcohol swab. The control group, MIN group, ITR group, VOR group, POS group, combined ITR and MIN group, combined VOR and MIN group, and combined POS and MIN group were injected with $10 \mathrm{ul}$ of saline, $10 \mathrm{ul} \mathrm{MIN}(12 \mu \mathrm{g} / \mathrm{ml}), 10 \mathrm{ul}$ ITR $(3 \mu \mathrm{g} / \mathrm{ml}), 10 \mathrm{ul}$ VOR $(0.75 \mu \mathrm{g} / \mathrm{ml}), 10 \mathrm{ul}$ POS $(1.5 \mu \mathrm{g} /$ $\mathrm{ml}), 10 \mathrm{ul}$ of mixed solution of MIN $(12 \mu \mathrm{g} / \mathrm{ml})$ and ITR 
$(3 \mu \mathrm{g} / \mathrm{ml}), 10 \mathrm{ul}$ of a mixture of MIN $(12 \mu \mathrm{g} / \mathrm{ml})$ and VOR $(0.75 \mu \mathrm{g} / \mathrm{ml})$, and $10 \mathrm{ul}$ of mixed solution of MIN $(12 \mu \mathrm{g} /$ $\mathrm{ml})$ and $\mathrm{POS}(1.5 \mu \mathrm{g} / \mathrm{ml})$, respectively. The larvae survival was recorded daily for 6 days. The survival curve of larvae was analysed by Kaplan-Meier method, and the difference was determined by Mantel Cox test. $P<0.05$ was considered statistically significant.

Larval tissues were collected, fixed in $10 \%$ neutral formalin and then dehydrated using an ethanol gradient. Thereafter, tissue samples were embedded in paraffin and xylene, sectioned at a thickness of $8 \mu \mathrm{m}$ and stained with hematoxylin and eosin (HE). Finally, stained sections were evaluated with FSX100 fluorescence microscopy at magnifications of $10 \times$ and $40 \times$.

\section{Abbreviations}

ITR: Itraconazole; VOR: Voriconazole; POS: Posaconazole; MIN: Minocycline; CNS: Central nervous system; MIC: Minimum inhibitory concentration; 1,3BDG: 1,3-beta-D-glucan; FICl: Fractional inhibitory concentration index; HE: Hematoxylin and eosin.

\section{Acknowledgments}

Thanks to Prof. Sybren de Hoog of the Dutch Fungal Biodiversity Centre (CBS) for the gift of six strains.

\section{Authors' contributions}

QL conceived and planned the study. YS contributed to the interpretation of the results. FY wrote the manuscript. All authors provided critical feedback and helped shape the research, analysis and manuscript. The authors read and approved the final manuscript.

\section{Funding}

This work was supported by Grant No. WJ2021 M261 from Health Commission of Hubei Province scientific research project and Grant No. 2019CFB567 from the Natural Science Foundation of Hubei Province.

\section{Availability of data and materials}

The data that support the findings of this study are available from the corresponding author upon reasonable request.

\section{Declarations}

Ethics approval and consent to participate

Not applicable.

\section{Consent for publication}

Not applicable.

\section{Competing interests}

The authors declare that there is no conflict of interest regarding the publication of this paper.

\section{Author details \\ 'Department of Dermatology, Shenzhen People's Hospital (The Second Clini- cal Medical College, Jinan University, The First Affiliated Hospital, Southern University of Science and Technology), Shenzhen 518020, Guangdong, China. ${ }^{2}$ Candidate Branch of National Clinical Research Center for Skin Diseases, Shenzhen, China. ${ }^{3}$ Department of Dermatology, Jingzhou Central Hospital, The Second Clinical Medical College, Yangtze University, Jingzhou, Hubei, China. ${ }^{4}$ Xiangyang Central Hospital, Affiliated Hospital of Hubei University of Arts and Science, Xiangyang, Hubei, China.}

Received: 19 Auqust 2021 Accepted: 9 December 2021 Published online: 12 January 2022

\section{References}

1. von Lilienfeld-Toal M, Wagener J, Einsele H, Cornely OA, Kurzai O. Invasive fungal infection. Dtsch Arztebl Int. 2019;116(16):271-8.

2. Sardi JCO, Scorzoni L, Bernardi T, Fusco-Almeida AM, Mendes Giannini MJS. Candida species: current epidemiology, pathogenicity, biofilm formation, natural antifungal products and new therapeutic options. J Med Microbiol. 2013:62(Pt 1):10-24.

3. Silva S, Negri M, Henriques M, Oliveira R, Williams DW, Azeredo J. Candida glabrata, Candida parapsilosis and Candida tropicalis: biology, epidemiology, pathogenicity and antifungal resistance. FEMS Microbiol Rev. 2012;36(2):288-305.

4. Cortez KJ, Roilides E, Quiroz-Telles F, Meletiadis J, Antachopoulos C, Knudsen T, et al. Infections caused by Scedosporium spp. Clin Microbiol Rev. 2008;21(1):157-97.

5. Bouchara JP, Papon N. Scedosporium apiospermum. Trends Microbiol. 2019;27(12):1045-6.

6. Allen D, Wilson D, Drew R, Perfect J. Azole antifungals: 35 years of invasive fungal infection management. Expert Rev Anti-Infect Ther. 2015;13(6):787-98.

7. Friedman DZP, Schwartz IS. Emerging fungal infections: new patients, new patterns, and new pathogens. J Fungi (Basel). 2019;5(3):67.

8. Lu Q, Sun Y, Tian D, Xiang S, Gao L. Effects of photodynamic therapy on the growth and antifungal susceptibility of Scedosporium and Lomentospora spp. Mycopathologia. 2017;182(11-12):1037-43.

9. Bentley S, Davies JC, Carr SB, Balfour-Lynn IM. Combination antifungal therapy for Scedosporium species in cystic fibrosis. Pediatr Pulmonol. 2020;55(8):1993-5.

10. Jenks JD, Seidel D, Cornely OA, Chen S, van Hal S, Kauffman C, et al. Voriconazole plus terbinafine combination antifungal therapy for invasive Lomentospora prolificans infections: analysis of 41 patients from the FungiScope(R) registry 2008-2019. Clin Microbiol Infect. 2020;26(6):784 e781-5.

11. Li H, Zhang C, Liu P, Liu W, Gao Y, Sun S. In vitro interactions between fluconazole and minocycline against mixed cultures of Candida albicans and Staphylococcus aureus. J Microbiol Immunol Infect. 2015;48(6):655-61.

12. Álvarez-Uría A, Guinea JV, Escribano P, Gómez-Castellá J, Valerio M, Galar $A$, et al. Invasive Scedosporium and Lomentosora infections in the era of antifungal prophylaxis: a 20-year experience from a single Centre in Spain. Mycoses. 2020;63(11):1195-202.

13. González GM, Fothergill AW, Sutton DA, Rinaldi MG, Loebenberg D. In vitro activities of new and established triazoles against opportunistic filamentous and dimorphic fungi. Med Mycol. 2005;43(3):281-4.

14. Meletiadis J, Meis JF, Mouton JW, Rodriquez-Tudela JL, Donnelly JP, Verweij PE. In vitro activities of new and conventional antifungal agents against clinical Scedosporium isolates. Antimicrob Agents Chemother. 2002;46(1):62-8.

15. Laihinen A, Rinne UK. Function of dopamine receptors in Parkinson's disease: prolactin responses. Neurology. 1986;36(3):393-5.

16. Kurakado S, Takatori K, Sugita T. Minocycline inhibits Candida albicans budded-to-Hyphal-form transition and biofilm formation. Jpn J Infect Dis. 2017;70(5):490-4.

17. de Oliveira LF, Jorge AO, Dos Santos SS. In vitro minocycline activity on superinfecting microorganisms isolated from chronic periodontitis patients. Braz Oral Res. 2006;20(3):202-6.

18. Schierholz JM, Pulverer G, Bach A, Wachol-Drebeck Z. In vitro activity of rifampin-minocyclin coating to Candida albicans. Crit Care Med. 1999;27(8):1691-3.

19. Wu Y, Grossman N, Totten M, Memon W, Fitzgerald A, Ying C, et al. Antifungal susceptibility profiles and drug resistance mechanisms of clinical Lomentospora prolificans isolates. Antimicrob Agents Chemother. 2020;64(11):e00318-20.

20. Jenks JD, Seidel D, Cornely OA, Chen S, van Hal S, Kauffman C, et al. Clinical characteristics and outcomes of invasive Lomentospora prolificans infections: analysis of patients in the FungiScope $\left({ }^{\circledR}\right)$ registry. Mycoses. 2020;63(5):437-42.

21. Gao L, Sun Y, Yuan M, Li M, Zeng T. In vitro and in vivo study on the synergistic effect of minocycline and azoles against pathogenic fungi. Antimicrob Agents Chemother. 2020;64(6):e00290-20. 
22. Cortez KJ, Roilides E, Quiroz-Telles F, Meletiadis J, Antachopoulos C, Knudsen T, et al. Infections caused by spp. Clin Microbiol Rev. 2008;21(1):157-97.

23. Nesky MA, Colin MDE, Peacock JE. Pseudallescheria boydii brain abscess successfully treated with Voriconazole and surgical drainage: case report and literature review of central nervous system Pseudallescheriasis. Clin Infect Dis. 2000;3:673-7.

24. Scott G, Zetterberg H, Jolly A, Cole JH, Simoni SD, Jenkins PO, et al. Minocycline reduces chronic microglial activation after brain trauma but increases neurodegeneration. Brain. 2018;141(2):459-71.

25. Daulatzai MA. Pharmacotherpy and Alzheimer's disease: the M-drugs (melatonin, minocycline, Modafinil, and Memantine) approach. Curr Pharm Des. 2016;22(16):2411-30.

26. Gilgado F, Cano J, Gené J, Guarro J. Molecular phylogeny of the Pseudallescheria boydii species complex: proposal of two new species. J Clin Microbiol. 2005:43(10):4930-42.

27. Odds FC. Synergy, antagonism, and what the chequerboard puts between them. J Antimicrob Chemother. 2003;52(1):1.

\section{Publisher's Note}

Springer Nature remains neutral with regard to jurisdictional claims in published maps and institutional affiliations.

- fast, convenient online submission

- thorough peer review by experienced researchers in your field

- rapid publication on acceptance

- support for research data, including large and complex data types

- gold Open Access which fosters wider collaboration and increased citations

- maximum visibility for your research: over $100 \mathrm{M}$ website views per year

At BMC, research is always in progress.

Learn more biomedcentral.com/submissions 